\title{
Effects of Bone Morphogenic Proteins on Neural Precursor Cells and Regulation during Central Nervous System Injury
}

\author{
Jennifer K. Sabo Trevor J. Kilpatrick Holly S. Cate \\ Centre for Neuroscience and Florey Neuroscience Institutes, The University of Melbourne, Parkville, Vic., Australia
}

\section{Key Words}

Central nervous system • Bone morphogenic protein •

Neural precursor cell $\cdot$ Oligodendrocyte $\cdot$ Astrocyte standing of the function of endogenous BMP regulation is important for determining how modulation of BMP signalling could improve repair following CNS injury.

Copyright ๑ 2009 S. Karger AG, Basel

Bone morphogenic proteins (BMPs) comprise the largest subclass of the transforming growth factor- $\beta$ (TGF- $\beta$ ) superfamily of cytokines and were originally identified for their roles in early bone formation [1] and gastrulation [2]. During nervous system development, BMPs have important actions in promoting dorsal identity, regulation of cell proliferation and regulation of cell fate $[3,4]$. Although less well studied, there is also evidence that BMPs modulate the production of oligodendrocytes, neurons and astrocytes from neural precursor cells (NPCs) in the adult central nervous system (CNS). In addition, there are increasing reports of regulation of BMPs following brain and spinal cord injury in rodents, although the functional consequences of this regulation are not completely clear. Given the functions of BMPs in the healthy CNS, modulation of signalling during CNS injury could directly affect the numbers and types of cells produced during critical periods following injury and impact on the repair process. Here, we review evidence for the roles of BMPs in precursor cell proliferation and differentiation in the CNS during late embryonic brain

\section{KARGER}

Fax +4161306 1234 E-Mail karger@karger.ch www.karger.com

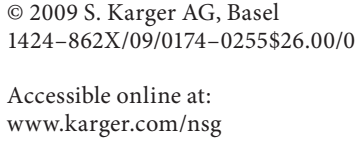

Holly S. Cate

Centre for Neuroscience, The University of Melbourne

Melbourne, Vic. 3010 (Australia)

Tel. +61 38344 7318, Fax +61 393470446

E-Mail hcate@unimelb.edu.au 


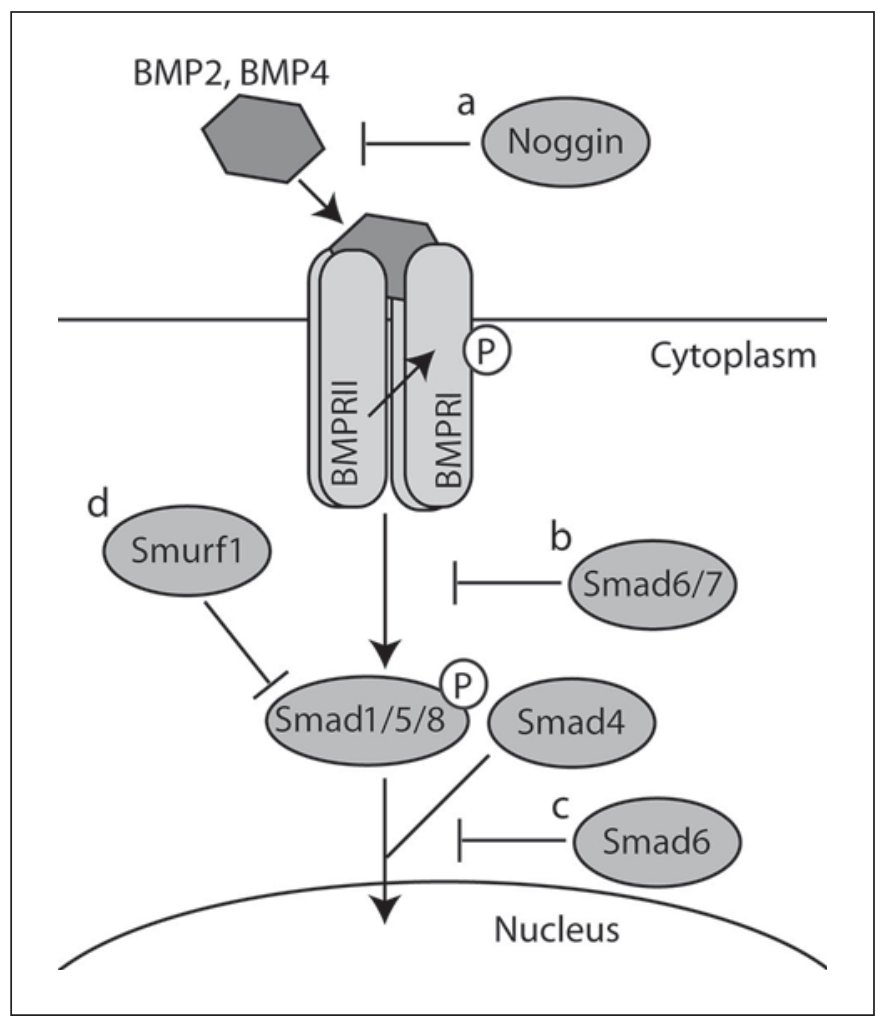

Fig. 1. Simplified illustration of the BMP signalling pathway. $\mathrm{BMP} 2$ and BMP4 bind to the BMP receptors BMPRI and BMPRII. $B$ phosphorylates and activates BMPRI, which phosphorylates receptor-activated SMADs (i.e. SMAD1/5/8). Phosphorylated receptor-activated SMADs associate with the common partner SMAD (i.e. SMAD4) and enter the nucleus, where they regulate gene expression. BMP signalling can be inhibited in many ways including: (a) binding BMPs by extracellular antagonists (e.g. noggin) [12]; (b) blocking phosphorylation of receptor-activated SMADs by inhibitory SMADs (e.g. SMAD6 and SMAD7) [8]; (c) competition with SMAD4 for binding with SMAD1/5/8 [13], or (d) ubiquitination and degradation of receptor-activated SMADs [14].

development and in the postnatal brain, predominantly in rodents. We will also discuss recent work that identifies regulation of BMP signalling following CNS injury.

\section{BMP Signalling}

There are more than 20 members of the BMP family and they are classified into 5 different subgroups [3]. BMP2 and BMP4, which share approximately $92 \%$ sequence homology [5] and comprise the Drosophila decapentaplegic gene product group (Dpp) of BMPs, signal through heteromeric serine/threonine kinase recep- tors composed of 2 type I signal-transducing receptors (BMPRIa and BMPRIb) and 1 type II (BMPRII) ligandbinding receptor [3]. Every BMP family member has a cysteine-rich $\mathrm{C}$-terminus involved in cysteine knot formation and homodimerisation [6]. The BMPs are secreted proteins produced in a precursor form characterised by the presence of a signal peptide, pro-domain and mature peptide [6]. Proteolytic processing and glycosylation of the mature peptide precede formation of C-terminal heterodimers or homodimers which are largely hydrophobic [6].

The BMP signalling pathway is well defined and is regulated by extracellular and intracellular antagonists (fig. 1). Upon BMP2 and BMP4 binding to the type I/type II heteromeric receptor complex, the type II receptor phosphorylates via its serine/threonine kinase domain the glycine-serine (GS) domain of the type I receptor, which in turn phosphorylates receptor-activated SMADs (i.e. SMAD1, SMAD5, and SMAD8) [for review, see 7, 8]. Each phosphorylated SMAD then forms a heteromeric complex with SMAD4, and the complex is translocated to the nucleus to interact with transcription factors [8]. SMAD1 has also been shown to interact with the glial differentiation signal, STAT (signal transducer and activator of transcription), through the transcriptional co-activator p300 [9]. In addition, BMP4 treatment of embryonic cortical NPCs can activate a distinct non-SMAD-mediated pathway to facilitate astroglial differentiation and STAT activation, in which the serine-threonine kinase FKB12/rapamycin-associated protein (FRAP) associates with STAT3 to induce differentiation [10].

The actions of the BMPs are regulated by extracellular antagonists and intracellular inhibitory mechanisms. Extracellular antagonists of BMPs include noggin, follistatin, follistatin-related protein (FSRP), chordin, chordin-like, cerberus, differential screening-selected gene aberrative in neuroblastomas (DAN), sclerostin, and gremlin [11]. The influences of noggin on BMP2 and BMP4 activity are well characterised. Noggin is a specific inhibitor of BMP2, BMP4 and, to a lesser extent, BMP7, and blocks signalling by preventing BMPs from binding to their receptors [12]. The interaction between BMP4 and noggin is tightly regulated, with noggin binding to BMP4 with picomolar affinity [12]. Signalling initiated by BMPs can also be inhibited intracellularly by inhibitory SMADs, or I-SMADs [8]. The two I-SMADs, SMAD6 and SMAD7, inhibit BMP and TGF- $\beta$ signalling by preventing phosphorylation of both receptor-activated SMADs and SMAD4 [8]. In addition, there has also been some evidence that SMAD6 can compete with 
SMAD4 to form a heteromeric complex with functionally active SMAD1 [13]. Ubiquitination and subsequent proteasomal degradation have been identified as an additional mechanism for intracellular inhibition of SMAD signalling $[14,15]$. The SMAD ubiquitination regulatory factor (Smurf)-1, a member of the Hect E3 ligase family, mediates the degradation of SMAD1 and SMAD5 [14], or alternatively Smurf1 is exported from the nucleus as a complex with SMAD6 or SMAD7 to mediate ubiquitination and degradation of BMPRIb [16].

\section{Expression of BMPs in the CNS}

The 3 BMP receptors, BMPRIa, BMPRIb and BMPRII, are expressed in the mouse brain beginning at embryonic day (E)12 [17]. The different mRNA expression patterns of the receptors reveal that BMPRIa is more broadly expressed in the developing brain in contrast to BMPRIb and BMPRII, which are restricted to the olfactory epithelium and ventricular zone, respectively [17]. As embryogenesis proceeds, the expression of the three receptors converges to the subventricular zone (SVZ) prior to cortical gliogenesis [17]. The BMP receptors can function alone or in combination with activin receptors [3], and this could explain why they initially have diverse expression profiles. Expression of BMP2 is localised to the cortex and ventricular zone, but is absent in corpus callosum and its expression is decreased in the subcortical white matter during development [18]. The expression level of BMP2 is low compared to BMP4, which is highly expressed in the SVZ [19]. In the rat spinal cord at embryonic day 14, BMP4 protein is expressed at high levels in the dorsal column and at lower levels in the ventral column and in cells forming the central canal [20].

In the adult, the expression of BMPs has been associated within the region of the SVZ. Both BMP2 and BMP4 and their receptors are expressed in the cells of the healthy adult mouse SVZ [21,22], although BMP2 is expressed at significantly lower levels [19]. Three classes of cells, commonly known as A, B and C cells, are present in the SVZ and contribute to an interrelated lineage pathway $[23,24]$. The B cells are astrocytes, immunoreactive for glial fibrillary acidic protein (GFAP) and are identified as selfrenewing neural stem cells $[23,24]$. The $\mathrm{C}$ cells are proliferative precursors and form clusters adjacent to A cells, which form chains of neuroblasts [23, 24]. These neuroblasts, which are ensheathed by B cells, migrate via the rostral migratory stream to the distant olfactory bulb where they commence their differentiation into neuronal subtypes [23-26]. Both BMP2 and BMP4 are specifically expressed in B/C cells, whereas BMPRIa and BMPRII are expressed in all 3 classes of SVZ cells. In contrast, BMPRIb is only expressed in A cells [21].

\section{Effects of BMPs in the Embryonic CNS}

The BMPs function in early embryonic development, most notably in dorsoventral patterning of the neural tube, has been reviewed elsewhere [2]. In this review, we will focus on the responses evoked by BMPs in embryonic CNS cells during cortical neurogenesis.

There is strong evidence that BMPs decrease proliferation in embryonic cell cultures [19, 27, 28]. In mouse neuroepithelial cultures, BMP2 decreased the number of MAP2+ neurons by inhibiting the proliferation of their progenitors [27]. Exogenous BMP2 also decreases proliferation in mouse SVZ progenitor cells [19]. Similarly, Mathieu et al. [28] showed that adult mouse endothelial cells produce BMP2 and BMP4 and co-culture with endothelial cells decreased the proliferation of E14.5 mouse NPCs in vitro. In addition, application of noggin to co-cultures of endothelial and NPCs significantly increased proliferation [28]. These findings suggest that BMPs inhibit the proliferation of embryonic cortical cells and SVZ NPCs.

As well as affecting their proliferation, BMPs influence the generation of glia from embryonic precursor cells. The BMPs promote astroglial differentiation of a variety of embryonic brain cells $[18,19,29,30]$. Specifically, BMP2 promoted astroglial differentiation of cells cultured from rat cerebral cortex [18]. Consistent with this, Gross et al. [19] found that exogenous BMP2 promoted astroglial differentiation of precursor cells isolated from mouse SVZ. In E18.5 mouse ganglionic eminence cultures, BMP4 promoted astroglial differentiation, while the BMP antagonist, noggin, inhibited astroglial differentiation [29]. In E14.5 mouse striatum NPCs infected with $\beta$-catenin retrovirus, which induces expression of BMPs, application of noggin also inhibited astroglial differentiation [30]. In contrast to promoting astroglial differentiation, BMPs have been reported to inhibit oligodendroglial differentiation $[18,30]$. Oligodendroglial differentiation was increased by noggin treatment of rat cerebral cortical cell cultures [18] and of NPCs from embryonic mouse striatum infected with $\beta$-catenin retrovirus [30].

The mechanisms underlying the effects of BMP signalling on glial lineage commitment were examined by Samanta et al. [31]. This study identified an association 
with BMP and early oligodendrocyte transcription factors, Olig1 and Olig2, in embryonic mouse NPCs and elucidated a molecular mechanism for the inhibition of oligodendrogliogenesis by BMP4 [31]. Inhibitors of differentiation (Ids), dominant negative inhibitors of basic helix-loop helix neurogenic transcription factors, are known to be major target genes for BMP/SMAD signalling $[32,33]$. Treatment with BMP4 or overexpression of Id 2 or Id 4 in E17 mouse NPCs reduced the percentage of 2',3'-cyclic nucleotide 3'-phosphodiesterase (CNPase) and myelin basic protein (MBP)-positive oligodendrocytes, while increasing the percentage of astrocytes expressing GFAP [31]. Using co-immunoprecipitation, these authors confirmed the interaction of the Olig and Id proteins. They found BMP4 treatment of NPCs resulted in the displacement of Olig1 and Olig2 from the nucleus, where they normally reside as transcription factors, to the cytoplasm and suggested a mechanism whereby the Ids are acting as a downstream effector of $\mathrm{BMP}$ signalling to inhibit oligodend roglial cell commitment.

The BMPs also negatively regulate the production of oligodendrocytes in the embryonic spinal cord. In embryonic rat spinal cord cultures, Miller et al. [20] showed that increasing concentrations of BMP4 decreased the number of $\mathrm{A} 2 \mathrm{~B} 5+$ oligodendrocyte progenitor cells (OPCs) and dramatically reduced $\mathrm{O} 4+$ premyelinating oligodendrocytes at E14, when OPCs appear in the spinal cord for the first time [34]. Oligodendrocyte production commences at E5.5 in the chick spinal cord [35], and when BMP4 was exogenously applied to E4.5 medial and ventral neural tube explants, the number of $\mathrm{O} 4+$ oligodendrocytes was decreased after 7 days in vitro [36]. The BMP4 treatment of E5 ventral neuroepithelial explants of the chick spinal cord also inhibited sonic hedgehog (Shh)induced oligodendrogliogenesis, but failed to fully inhibit the process once it had been initiated at E6 [36]. In addition, cells overexpressing BMP2, when engrafted at E33.5 in the developing ventral spinal cord, reduced the expression of Olig2 at E7-7.5 [36]. Exogenous BMP4 also significantly decreased the number of Olig2+ nuclei in E5 ventral explant cultures in vitro [36]. Similar to the effect of BMP in rodent and chick models, there was reduced generation of oligodendroglia in response to the treatment of human fetal spinal cord NPCs with BMP4 [37]. These results support an inhibitory role for BMPs in the production of oligodendrocytes in the developing spinal cord.

The BMPs have also been observed to influence the neuronal differentiation of embryonic precursor cells. In cortical precursor cells, BMP2 increased neuronal differentiation in low- and moderate-density cultures [18]. In contrast, BMP2 decreased neuronal differentiation of SVZ precursor cells [19]. By utilising total live cell counts Nakashima et al. [27] clearly showed that BMP2 altered the fate of embryonic neuroepithelial cells from a neuronal to an astroglial lineage in vitro [27]. In conclusion, BMPs modulate the production of neurons, but the specific effect, whether promotion or inhibition, appears to be dependent on the origin of the precursor cells.

\section{Roles of BMPs in the Postnatal CNS}

In contrast to their roles in embryonic development, the actions of the BMPs are not as well characterised in the adult. Existing evidence in the adult has predominantly implicated BMPs in promoting astrogliosis and inhibiting oligodendrogliogenesis, which is consistent with their role in embryonic development. The role of BMPs in neurogenesis is less clear.

The BMPs affect the proliferation and lineage commitment of NPCs in the adult rodent SVZ. Proliferation is decreased in co-cultures of adult mouse SVZ NPCs and endothelial cells, which are an innate source of BMPs [28]. In these studies, cells were co-cultured in the presence of the mitogens epidermal growth factor (EGF) and fibroblast growth factor (FGF). In another study, Samanta et al. [38] generated transgenic mice where the BMP receptor, BMPRIa, was conditionally deleted in Olig1 progenitors. They found that SVZ NPCs in conditional knockout (CKO) mice had a shortened cell cycle compared to wild-type (WT) mice and that this contributed to an increased number of oligodendroglia [38]. These investigations also found an increase in the number of cortical CNPase+ and myelinating $\mathrm{MBP}+$ oligodendrocytes at P20 in the CKO mice compared to WT [38]. These results support the conclusion that BMP signalling alters NPC proliferation and oligodendroglial production from adult NPCs.

Studies using transgenic overexpression of BMP4 have also been used to describe the effects of BMPs on oligodendroglial and astroglial production in vivo. The BMP4 was transgenically overexpressed in the brains of mice under the control of a neuron-specific enolase promoter from E16 through to adulthood [39]. Transgenic overexpression of BMP4 decreased the number of glutathione S-transferase $\pi$ (GST-Pi)-positive oligodendrocytes in the neocortex and CNPase+ oligodendrocytes in the hippocampus of the adult [39]. In the brains of these mice, 
astrocyte production was significantly increased, as shown by quantification of $\mathrm{S} 100 \mathrm{~B}+$ astrocytes in the neocortex. These studies suggest that BMP promotes generation of astrocytes and decreases oligodendrocyte production in vivo.

Studies using in vitro oligodendrocyte progenitor cell (OPC) cultures have highlighted the direct effect of BMPs on oligodendrocyte lineage progression. In figure 2 , we have illustrated the effects of BMP on the various stages of the oligodendrocyte lineage, which are characterised by the presence of different antigens. A comprehensive study by Grinspan et al. [40] reported the specific effects of BMPs on 3 stages of oligodendrocyte lineage in vitro: pre-progenitors, progenitors and myelinating oligodendrocytes. Grinspan et al. [41] originally identified the oligodendrocyte pre-progenitor (pre-OPC), which is isolated from the rat forebrain immediately following birth and which does not express GFAP or A2B5, but which will differentiate into an OPC with platelet-derived growth factor (PDGF) treatment. Addition of BMP2 and BMP4 to oligodendrocyte pre-progenitors resulted in cells expressing GFAP and polysialylated neural cell adhesion molecule (PSA-NCAM) with a novel astrogliallike phenotype [40]. In contrast, when OPCs were exposed to BMP2 and BMP4, the cells acquired a stellate, type 2 astrocyte phenotype and expressed A2B5 and GFAP. Addition of BMP2 and BMP4 to OPC cultures rarely resulted in cells that expressed $\mathrm{O} 4$, galactocerebroside (GalC) or MBP, which are markers of more mature oligodendrocytes. Myelinating GalC+ oligodendrocytes were found to be unresponsive to BMP treatment, reflecting the selectivity of BMPs for specific stages of oligodendrocyte lineage development [40]. This study highlights the inhibitory role of BMPs on oligodendrocyte development, in particular, on the differentiation of pre-OPCs and OPCs into premyelinating oligodendrocytes.

The BMPs can also influence the differentiation of rat optic nerve OPCs (previously referred to as oligodendrocyte type 2 astrocyte (O-2A) progenitor cells), which can be differentiated into oligodendrocytes or type 2 astrocytes in vitro $[42,43]$. These OPCs have been observed to differentiate into oligodendrocytes in the absence of serum, whereas they differentiate into type 2 astrocytes in its presence [42]. Exogenous application of BMPs can reproduce the effects of serum in culture by inducing astroglial differentiation of OPCs $[44,45]$. In OPC cultures without serum, BMP2, 4 and 7 induced GFAP+ astroglial differentiation, while suppressing oligodendrocyte differentiation [44]. Interestingly, even after differentiating to type 2 astrocytes following serum exposure, OPCs

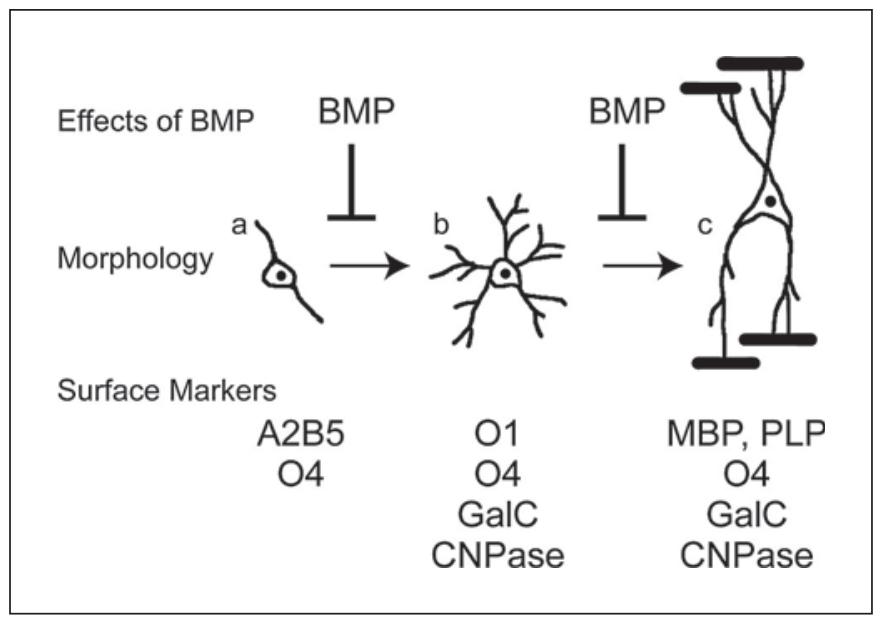

Fig. 2. Cartoon illustrating three stages of oligodendroglial lineage cells: (a) oligodendrocyte progenitor cell (OPC); (b) premyelinating oligodendrocyte, and (c) myelinating oligodendrocyte. Modified from Woodruff et al. [48]. Surface markers that are commonly used to classify these stages are shown. BMP has been shown to inhibit the differentiation of OPCs to premyelinating oligodendrocytes [40] and to inhibit the maturation of premyelinating oligodendrocytes $[50,51]$.

can give rise to neurons, astrocytes, and oligodendrocytes in the presence of bFGF [45]. On the one hand, this study showed that BMP2 and BMP4 could induce the type 2 astrocyte phenotype in postnatal rat optic nerve OPC cultures in the absence of serum (similar to results obtained from forebrain OPCs [40]). On the other hand, the addition of BMP2 and BMP4 did not prevent the OPCs from responding to bFGF and differentiating into neurons and oligodendrocytes [45]. Kondo and Raff [46] found that BMP4 and BMP receptors, BMPRIa and BMPRII, are expressed in OPCs and oligodendrocytes from rat optic nerve, whereas noggin is expressed only in OPCs and type 1 astrocytes isolated from this site [46]. Unlike type 2 astrocytes, type 1 astrocytes are A2B5-negative [42]. Silencing of noggin by transfecting OPCs with siRNA induced type 2 astroglial differentiation in lowserum conditions (2.5\% FCS), whereas noggin overexpression in high-serum conditions (5\% FCS) blocked the production of type 2 astrocytes [46]. Unlike in culture, there is a lack of compelling evidence that OPCs develop into type 2 astrocytes in vivo. Kondo and Raff [46] speculate that an endogenous source of noggin in OPCs could prevent the formation of type 2 astrocytes in vivo. The BMPs also regulate the astroglial differentiation of adult human OPCs. Gene profiling studies revealed that adult human A2B5+ OPCs express BMP2 and BMP7 and when 
BMP4 was exogenously applied to these cultures, there was an increase in the percentage of GFAP+/A2B5+ cells (i.e. type 2 astrocytes) [47]. From this cumulative evidence, OPCs are responsive to BMPs, which inhibit the oligodendroglial differentiation of OPCs and promote the generation of type 2 astrocytes.

In addition to the effects on oligodendrocyte differentiation, BMPs have also been implicated in the maturation of oligodendrocytes. Premyelinating oligodendrocytes are still immature and express GalC [48] and CNPase [49]. As they mature, oligodendrocytes continue to express CNPase [49] and express myelin markers such as proteolipid protein (PLP) and MBP [48]. Cheng et al. [50] showed that BMP2 and BMP4 directly inhibited the maturation of oligodendrocytes from the $\mathrm{O} 1+$ premyelinating phase into myelinating $\mathrm{MBP}+$ and CNPase+ oligodendrocytes amongst cells originally derived from OPCs isolated from the rat spinal cord. Exogenous BMP4 decreased the expression of CNPase, PLP and MBP in P1 rat forebrain premyelinating oligodendrocytes under differentiating conditions [51]. The effect of BMP on inhibition of maturation was dose-dependent [51]. In addition, BMP4 failed to inhibit myelination if myelin protein synthesis had already commenced [51]. These studies depict BMPs as negatively regulating myelin protein synthesis and consequently inhibiting the maturation of oligodendrocytes.

However, a very different conclusion was subsequently reached by See et al. [52] who used BMP receptor double knockout mice, which are functionally null for BMPRIa and BMPRIb in the neural tube, to study the effects of endogenous BMP signalling on astroglial and oligodendroglial differentiation in the spinal cord. Not surprisingly, they found a significant decrease in the number of GFAP+ astrocytes in the P0 mouse spinal cord [52]. However, in addition, they reported a decrease in MBP and PLP expression in the spinal cord of the BMPR double $\mathrm{KO}$ mice, and there was a concurrent and significant decrease in the numbers of GalC+ oligodendrocytes. On the other hand, the BMPR double KO had no effect on emergence and the overall number or proliferation of OPCs [52]. This is in contrast to in vitro studies reporting that BMPs inhibit oligodendrocyte lineage development $[40,50,51]$, and See et al. [52] suggest that BMP receptor activation is required for oligodendrocyte differentiation and myelin production.

In addition to their roles in gliogenesis in the postnatal CNS, BMPs play an important but less understood role in adult neurogenesis. Signalling initiated by BMPs is active in the adult SVZ and hippocampus as demonstrated by phosphorylated SMAD 1/5/8 immunoreactivity in vivo and in vitro $[22,53]$. However, reports of the effects of BMP signalling on neurogenesis are conflicting. Lim et al. [21] found that exogenous BMP2 and BMP4 reduced the production of neurons expressing $\beta I I I$ tubulin (i.e. Tuji+) more than 10-fold in co-cultures of astrocytes and SVZ type B and C cells. Conversely, noggin increased the production of neurons by $20 \%$ in both these co-cultures and also in SVZ aggregates where it also inhibited astroglial differentiation. In a series of in vivo studies, Lim et al. [21] found that overexpression of BMP7 decreased the endogenous production of neuroblasts and that noggin overexpression by adenoviral injection increased neuronal differentiation of healthy donor SVZ cells grafted into the striatum. In essence, this study suggests that BMP inhibits neurogenesis. In contrast, Colak et al. [22] have shown that BMP signalling is both an important initiator of neurogenesis and a suppressor of oligodendrogliogenesis. To disrupt BMP signalling, they used a genetic approach whereby they created a conditional deletion of SMAD4 driven by the glutamate transporter (GLAST) promoter that is specific for SVZ neural stem cells and astrocytes [22]. Immunostaining revealed that SMAD4 deletion significantly decreased the number of DCX+ neuroblasts and resulted in NPC-derived cells migrating from the SVZ to the corpus callosum where they differentiated in oligodendrocytes [22]. Colak et al. [22] also showed that neurogenesis was restored when Olig2 function was suppressed by viral injection of the antagonist Olig2VP16 [54]. Noggin infusion into the lateral ventricle of WT mice was shown to significantly increase the number of Olig2 + cells and to decrease numbers of DCX+ neuroblasts in the SVZ [22]. This study therefore suggested that BMP promotes neurogenesis.

There is also evidence of a role for BMP signalling in neurogenesis in the hippocampus, another area of ongoing neurogenesis in the adult brain. Bonaguidi et al. [53] showed that neuronal differentiation was reduced by the addition of BMP4 to adult hippocampal neurospheres. They also found that transgenic overexpression of noggin under the control of the neuron-specific enolase promoter in the hippocampus increased GFAP+/BrdU+ cells, which they suggest are neural stem cells and could represent a niche for adult neurogenesis [53]. Taken together, these results demonstrate that BMP signalling plays a role in neurogenesis; however, the data suggest that BMP could have differing effects dependent on the cell niche of the precursor cells and upon the context in which it is provided. 
Table 1. Summary of studies identifying regulation of BMPs during CNS injury

\begin{tabular}{|c|c|c|c|}
\hline $\begin{array}{l}\text { BMP family } \\
\text { member }\end{array}$ & Response in CNS injury & Potential role & Reference \\
\hline \multirow[t]{2}{*}{ BMP2 } & Upregulated in EB-induced lesion in rat thoracic spinal cord & Schwann cell differentiation & 66 \\
\hline & Upregulation in the rat and mouse spinal cord following contusion and stab injury & Astrogliosis & $50,60,61$ \\
\hline \multirow[t]{6}{*}{ BMP4 } & Upregulated in EB-induced lesion in rat thoracic spinal cord & Schwann cell differentiation & 66 \\
\hline & Strongly upregulated in the lumbar spinal cord of demyelinated EAE mice & Inflammatory response in EAE & 57 \\
\hline & Upregulated in OPCs during remyelination in a rat EB model of demyelination & Oligodendroglial differentiation & 55 \\
\hline & Upregulated in a lysolecithin-induced lesion in the rat spinal cord & Glial scar formation & 56 \\
\hline & Detected in human brain chronic MS lesion & Inflammatory response & 58 \\
\hline & Upregulation in the rat and mouse spinal cord following contusion and stab injury & Astrogliosis & 50,61 \\
\hline BMP5 & Detected in human brain chronic MS lesion & Inflammatory response & 58 \\
\hline \multirow[t]{2}{*}{ BMP6 } & Upregulated in the lumbar spinal cord of demyelinated EAE mice & Not proposed & 57 \\
\hline & Upregulated in the rat brain following traumatic brain injury induced by contusion & Reactive astrogliosis & 67 \\
\hline \multirow[t]{4}{*}{ BMP7 } & Upregulated in the lumbar spinal cord of demyelinated EAE mice & Not proposed & 57 \\
\hline & Upregulated in a lysolecithin-induced myelin lesion in the rat spinal cord & Glial scar formation & 56 \\
\hline & Upregulation in the rat and mouse spinal cord following contusion and stab injury & Astrogliosis & 59,61 \\
\hline & Upregulated in the brain following middle cerebral artery occlusion in rats & Neuroregeneration & 63 \\
\hline
\end{tabular}

\section{Regulation of BMPs during CNS Injury}

Given the regulatory role of BMPs upon cell lineage commitment in the healthy adult, it is not surprising that a role is emerging for BMPs in CNS injury. Here, we will discuss the growing evidence for regulation of the expression of BMPs in the context of CNS injury. There is also some evidence that modulation of BMP signalling alters the repair process following CNS injury. Table 1 summarises the data on regulation of BMPs during CNS injury and the potential function of BMPs in this context. Further investigation into the function of BMPs during CNS injury could provide us with insights that would enable us to enhance the repair process and has been a focus of work in our laboratory.

There is evidence that BMP levels are elevated in demyelinating lesions in the CNS. Expression of BMP4 was increased specifically in OPCs within demyelinated lesions induced by ethidium bromide in the caudal cerebellar peduncle of rats, but subsequently is undetectable upon complete remyelination [55]. This suggests that there may be a beneficial role for BMP4 in oligodendrocyte differentiation as the majority of OPCs become oligodendrocytes during the remyelination process [55]. In lysolecithin-induced demyelinated lesions in the adult rat spinal cord, the expression profiles of BMP4 and BMP7 were increased and associated with glial scar formation [56]. In an EAE model, BMPs 4, 6 and 7 were upregulated in the spinal cord with BMP4 mRNA and protein expression being most abundant and increasing with disease severity [57]. The regulation of BMP4 could be associated with the inflammatory response in EAE as it was predominately co-localised with microglia/macrophages [57]. Demyelinating lesions in humans have also been shown to express BMPs. Both BMP4 and BMP5 were found to be expressed in chronic MS lesions identified in postmortem brain tissue [58]. Taken together, these studies suggest that BMPs could have important functions within lesions during CNS myelin injury.

The BMPs have also been found to be upregulated in spinal cords that were injured by compression or knife cut [50,59-61], and this upregulated expression is associated with an increase in astrogliosis $[59,60]$. In addition, embryonic NPCs virally transfected to express noggin have been transplanted into adult mouse spinal cord following compression injury and the transplanted cells have been observed to exhibit increased oligodendrocyte and neuronal differentiation, with concordant partial functional recovery [60]. On the other hand, the transplantation of OPCs virally transfected to express noggin did not influence oligodendrocyte lineage commitment in rats following spinal cord injury [62]. These studies demonstrate that BMPs are involved in the cellular response to CNS injury; however, the effects of BMP inhibition on repair are not yet clear. 
There is also evidence that an increase in endogenous BMPs could regulate the glial response to penetrating injury to the mouse brain. Hampton et al. [61] used a functional noggin-blocking antibody to invoke increased endogenous production of BMPs in order to examine the relationship between OPCs and type 2 astrocytes in vitro and in vivo. In vitro, the noggin-blocking antibody resulted in the generation of type 2 astrocytes in cultures of cortical chondroitin sulphate proteoglycan (NG2)-positive OPCs isolated from rat [61]. These investigators also infused the noggin-blocking antibody into the brains of mice subjected to injury by knife cut, and found a significant increase in the number of double-positive NG2+GFAP+ cells, which exhibited characteristics of type 2 astrocytes [61]. This study suggests that BMPs may modulate the fate of OPCs during injury.

In rat models of experimental stroke, studies suggest BMP7, which is upregulated following stroke [63], could play a neuroprotective role when it is administered $24 \mathrm{~h}$ after injury [63-65]. Following middle cerebral artery occlusion (MCAO) in rats, BMP7 administered intracisternally improved neurological function as well as local cerebral blood flow and local cerebral glucose utilisation [64]. In another study, systemic delivery of BMP7 after MCAO decreased body asymmetry, based on an elevated body swing test, and improved locomotor activity [63]. In addition, Chou et al. [65] observed an increase in the number of BrdU+ cells in the lesioned cortex, SVZ and corpus callosum with BMP7 infusion after MCAO. In this study, the BrdU+ cells mainly co-labelled with the neuronal markers, NeuN or Nestin, which the authors suggest provided evidence of increased neuronal differentiation with BMP7 infusion following injury.

In conclusion, BMPs play key roles in affecting the proliferation and differentiation of adult neural precursor cells and the maturation and myelin production of oligodendroglia. Signalling initiated by BMP is modulated during CNS injury, potentially altering some or all of these processes. Currently, the roles of BMPs in the response to CNS injury are not well characterised, and require further investigation. In the meantime, potentially relevant information on possible functions can be gained from studies in healthy embryonic and adult CNS, although care is required in the extrapolation of these results, as even under these circumstances, as this review highlights, their effects are potentially highly context-dependent. Understanding the roles that BMP signalling plays during brain injury is critical to determine whether modulation of BMP signalling might promote repair responses.

\section{References}

1 Tsumaki N, Yoshikawa H: The role of bone morphogenetic proteins in endochondral bone formation. Cytokine Growth Factor Rev 2005;16:279-285.

$\checkmark 2$ Liu A, Niswander LA: Bone morphogenetic protein signalling and vertebrate nervous system development. Nat Rev Neurosci 2005; 6:945-954.

$\checkmark 3$ Mehler MF, Mabie PC, Zhang D, Kessler JA: Bone morphogenetic proteins in the nervous system. Trends Neurosci 1997;20:309-317.

$\checkmark 4$ Chen HL, Panchision DM: Concise review: bone morphogenetic protein pleiotropism in neural stem cells and their derivatives - alternative pathways, convergent signals. Stem Cells 2007;25:63-68.

5 Kingsley DM: The TGF- $\beta$ superfamily: new members, new receptors, and new genetic tests of function in different organisms. Genes Dev 1994;8:133-146.

6 Xiao YT, Xiang LX, Shao JZ: Bone morphogenetic protein. Biochem Biophys Res Commun 2007;362:550-553.

$\checkmark 7$ Massague J: TGF- $\beta$ signal transduction. Annu Rev Biochem 1998;67:753-791.
8 Itoh S, Itoh F, Goumans MJ, Ten Dijke P: Signaling of transforming growth factor- $\beta$ family members through Smad proteins. Eur J Biochem 2000;267:6954-6967.

9 Nakashima K, Yanagisawa M, Arakawa H, Kimura N, Hisatsune T, Kawabata M, Miyazono K, Taga T: Synergistic signaling in fetal brain by STAT3-Smad1 complex bridged by p300. Science 1999;284:479-482.

$>10$ Rajan P, Panchision DM, Newell LF, McKay RD: BMPs signal alternately through a SMAD or FRAP-STAT pathway to regulate fate choice in CNS stem cells. J Cell Biol 2003; 161:911-921.

11 Balemans W, Van Hul W: Extracellular regulation of BMP signaling in vertebrates: a cocktail of modulators. Dev Biol 2002;250: 231-250.

12 Zimmerman LB, De Jesus-Escobar JM, Harland RM: The Spemann organizer signal noggin binds and inactivates bone morphogenetic protein 4. Cell 1996;86:599-606.

13 Hata A, Lagna G, Massague J, HemmatiBrivanlou A: Smad6 inhibits BMP/Smad1 signaling by specifically competing with the Smad4 tumor suppressor. Genes Dev 1998; 12:186-197.
$>14$ Zhu H, Kavsak P, Abdollah S, Wrana JL, Thomsen GH: A SMAD ubiquitin ligase targets the BMP pathway and affects embryonic pattern formation. Nature 1999;400:687693.

15 Moren A, Imamura T, Miyazono K, Heldin $\mathrm{CH}$, Moustakas A: Degradation of the tumor suppressor Smad4 by WW and HECT domain ubiquitin ligases. J Biol Chem 2005; 280:22115-22123.

16 Murakami G, Watabe T, Takaoka K, Miyazono K, Imamura T: Cooperative inhibition of bone morphogenetic protein signaling by Smurf1 and inhibitory Smads. Mol Biol Cell 2003;14:2809-2817.

17 Zhang D, Mehler MF, Song Q, Kessler JA: Development of bone morphogenetic protein receptors in the nervous system and possible roles in regulating trkC expression. J Neurosci 1998;18:3314-3326.

18 Mabie PC, Mehler MF, Kessler JA: Multiple roles of bone morphogenetic protein signaling in the regulation of cortical cell number and phenotype. J Neurosci 1999;19:70777088 . 
19 Gross RE, Mehler MF, Mabie PC, Zang Z, Santschi L, Kessler JA: Bone morphogenetic proteins promote astroglial lineage commitment by mammalian subventricular zone progenitor cells. Neuron 1996;17:595-606.

20 Miller RH, Dinsio K, Wang R, Geertman R, Maier CE, Hall AK: Patterning of spinal cord oligodendrocyte development by dorsally derived BMP4. J Neurosci Res 2004;76:919.

21 Lim DA, Tramontin AD, Trevejo JM, Herrera DG, Garcia-Verdugo JM, Alvarez-Buylla A: Noggin antagonizes BMP signaling to create a niche for adult neurogenesis. Neuron 2000;28:713-726.

22 Colak D, Mori T, Brill MS, Pfeifer A, Falk S, Deng C, Monteiro R, Mummery C, Sommer L, Gotz M: Adult neurogenesis requires Smad4-mediated bone morphogenic protein signaling in stem cells. J Neurosci 2008;28: 434-446.

23 Doetsch F, Garcia-Verdugo JM, AlvarezBuylla A: Cellular composition and three-dimensional organization of the subventricular germinal zone in the adult mammalian brain. J Neurosci 1997;17:5046-5061.

24 Doetsch F, Caille I, Lim DA, Garcia-Verdugo JM, Alvarez-Buylla A: Subventricular zone astrocytes are neural stem cells in the adult mammalian brain. Cell 1999;97:703-716.

-25 Lois C, Alvarez-Buylla A: Long-distance neuronal migration in the adult mammalian brain. Science 1994;264:1145-1148.

-26 Doetsch F, Alvarez-Buylla A: Network of tangential pathways for neuronal migration in adult mammalian brain. Proc Natl Acad Sci USA 1996;93:14895-14900.

-27 Nakashima K, Takizawa T, Ochiai W, Yanagisawa $M$, Hisatsune T, Nakafuku M, Miyazono K, Kishimoto T, Kageyama R, Taga T: BMP2-mediated alteration in the developmental pathway of fetal mouse brain cells from neurogenesis to astrocytogenesis. Proc Natl Acad Sci USA 2001;98:5868-5873.

28 Mathieu C, Sii-Felice K, Fouchet P, Etienne O, Haton C, Mabondzo A, Boussin FD, Mouthon MA: Endothelial cell-derived bone morphogenetic proteins control proliferation of neural stem/progenitor cells. Mol Cell Neurosci 2008;38:569-577.

-29 Bonaguidi MA, McGuire T, Hu M, Kan L, Samanta J, Kessler JA: LIF and BMP signaling generate separate and discrete types of GFAP-expressing cells. Development 2005; 132:5503-5514

- 30 Kasai M, Satoh K, Akiyama T: Wnt signaling regulates the sequential onset of neurogenesis and gliogenesis via induction of BMPs. Genes Cells 2005;10:777-783.

- 31 Samanta J, Kessler JA: Interactions between ID and OLIG proteins mediate the inhibitory effects of BMP4 on oligodendroglial differentiation. Development 2004; 131:41314142 .

Effects of BMPs on Neural Precursor Cells and Regulation during CNS Injury
32 Hollnagel A, Oehlmann V, Heymer J, Ruther $\mathrm{U}$, Nordheim A: Id genes are direct targets of bone morphogenetic protein induction in embryonic stem cells. J Biol Chem 1999;274: 19838-19845.

33 Ying QL, Nichols J, Chambers I, Smith A: BMP induction of Id proteins suppresses differentiation and sustains embryonic stem cell self-renewal in collaboration with STAT3. Cell 2003;115:281-292.

34 Pringle NP, Richardson WD: A singularity of PDGF alpha-receptor expression in the dorsoventral axis of the neural tube may define the origin of the oligodendrocyte lineage. Development 1993;117:525-533.

35 Soula C, Danesin C, Kan P, Grob M, Poncet C, Cochard P: Distinct sites of origin of oligodendrocytes and somatic motoneurons in the chick spinal cord: oligodendrocytes arise from $\mathrm{Nkx} 2.2$-expressing progenitors by a Shh-dependent mechanism. Development 2001;128:1369-1379.

36 Mekki-Dauriac S, Agius E, Kan P, Cochard $\mathrm{P}$ : Bone morphogenetic proteins negatively control oligodendrocyte precursor specification in the chick spinal cord. Development 2002;129:5117-5130.

37 Weible MW 2nd, Chan-Ling T: Phenotypic characterization of neural stem cells from human fetal spinal cord: synergistic effect of LIF and BMP4 to generate astrocytes. Glia 2007;55:1156-1168.

- 38 Samanta J, Burke GM, McGuire T, Pisarek AJ, Mukhopadhyay A, Mishina Y, Kessler JA: Bmprla signaling determines numbers of oligodendrocytes and calbindin-expressing interneurons in the cortex. J Neurosci 2007; 27:7397-7407.

39 Gomes WA, Mehler MF, Kessler JA: Transgenic overexpression of BMP4 increases astroglial and decreases oligodendroglial lineage commitment. Dev Biol 2003;255: 164-177.

40 Grinspan JB, Edell E, Carpio DF, Beesley JS, Lavy L, Pleasure D, Golden JA: Stage-specific effects of bone morphogenetic proteins on the oligodendrocyte lineage. J Neurobiol 2000;43:1-17.

-41 Grinspan JB, Stern JL, Pustilnik SM, Pleasure D: Cerebral white matter contains PDGF-responsive precursors to O2A cells. J Neurosci 1990;10:1866-1873.

42 Raff MC, Miller RH, Noble M: A glial progenitor cell that develops in vitro into an astrocyte or an oligodendrocyte depending on culture medium. Nature 1983;303:390-396.

43 Raff MC: Glial cell diversification in the rat optic nerve. Science 1989;243:1450-1455.

44 Mabie PC, Mehler MF, Marmur R, Papavasiliou A, Song Q, Kessler JA: Bone morphogenetic proteins induce astroglial differentiation of oligodendroglial-astroglial progenitor cells. J Neurosci 1997;17:4112-4120.

45 Kondo T, Raff M: Oligodendrocyte precursor cells reprogrammed to become multipotential CNS stem cells. Science 2000;289: 1754-1757.
46 Kondo T, Raff MC: A role for noggin in the development of oligodendrocyte precursor cells. Dev Biol 2004;267:242-251.

47 Sim FJ, Lang JK, Waldau B, Roy NS, Schwartz TE, Pilcher WH, Chandross KJ, Natesan S, Merrill JE, Goldman SA: Complementary patterns of gene expression by human oligodendrocyte progenitors and their environment predict determinants of progenitor maintenance and differentiation. Ann Neurol 2006;59:763-779.

48 Woodruff RH, Tekki-Kessaris N, Stiles CD, Rowitch DH, Richardson WD: Oligodendrocyte development in the spinal cord and telencephalon: common themes and new perspectives. Int J Dev Neurosci 2001;19: 379-385.

49 Scherer SS, Braun PE, Grinspan J, Collarini E, Wang DY, Kamholz J: Differential regulation of the $2^{\prime}, 3^{\prime}$-cyclic nucleotide $3^{\prime}$-phosphodiesterase gene during oligodendrocyte development. Neuron 1994;12:1363-1375.

50 Cheng X, Wang Y, He Q, Qiu M, Whittemore SR, Cao Q: Bone morphogenetic protein signaling and Olig1/2 interact to regulate the differentiation and maturation of adult oligodendrocyte precursor cells. Stem Cells 2007;25:3204-3214.

51 See J, Zhang X, Eraydin N, Mun SB, Mamontov P, Golden JA, Grinspan JB: Oligodendrocyte maturation is inhibited by bone morphogenetic protein. Mol Cell Neurosci 2004; 26:481-492.

52 See J, Mamontov P, Ahn K, Wine-Lee L, Crenshaw EB 3rd, Grinspan JB: BMP signaling mutant mice exhibit glial cell maturation defects. Mol Cell Neurosci 2007;35:171-182.

53 Bonaguidi MA, Peng CY, McGuire T, Falciglia G, Gobeske KT, Czeisler C, Kessler JA: Noggin expands neural stem cells in the adult hippocampus. J Neurosci 2008;28: 9194-9204.

-54 Mizuguchi R, Sugimori M, Takebayashi H, Kosako H, Nagao M, Yoshida S, Nabeshima Y, Shimamura K, Nakafuku M: Combinatorial roles of Olig2 and Neurogenin2 in the coordinated induction of pan-neuronal and subtype-specific properties of motoneurons. Neuron 2001;31:757-771

55 Zhao C, Fancy SP, Magy L, Urwin JE, Franklin RJ: Stem cells, progenitors and myelin repair. J Anat 2005;207:251-258

56 Fuller ML, DeChant AK, Rothstein B, Caprariello A, Wang R, Hall AK, Miller RH: Bone morphogenetic proteins promote gliosis in demyelinating spinal cord lesions. Ann Neurol 2007;62:288-300.

57 Ara J, See J, Mamontov P, Hahn A, Bannerman P, Pleasure D, Grinspan JB: Bone morphogenetic proteins 4,6 , and 7 are up-regulated in mouse spinal cord during experimental autoimmune encephalomyelitis. J Neurosci Res 2008;86:125-135. 
-58 Deininger M, Meyermann R, Schluesener H: Detection of two transforming growth factor- $\beta$-related morphogens, bone morphogenetic proteins -4 and -5 , in RNA of multiple sclerosis and Creutzfeldt-Jakob disease lesions. Acta Neuropathol 1995;90:76-79.

-59 Setoguchi T, Yone K, Matsuoka E, Takenouchi H, Nakashima K, Sakou T, Komiya S, Izumo S: Traumatic injury-induced BMP7 expression in the adult rat spinal cord. Brain Res 2001;921:219-225.

-60 Setoguchi T, Nakashima K, Takizawa T, Yanagisawa M, Ochiai W, Okabe M, Yone K, Komiya S, Taga T: Treatment of spinal cord injury by transplantation of fetal neural precursor cells engineered to express BMP inhibitor. Exp Neurol 2004;189:33-44.
61 Hampton DW, Asher RA, Kondo T, Steeves JD, Ramer MS, Fawcett JW: A potential role for bone morphogenetic protein signalling in glial cell fate determination following adult central nervous system injury in vivo. Eur J Neurosci 2007;26:3024-3035.

62 Enzmann GU, Benton RL, Woock JP, Howard RM, Tsoulfas P, Whittemore SR: Consequences of noggin expression by neural stem, glial, and neuronal precursor cells engrafted into the injured spinal cord. Exp Neurol 2005;195:293-304.

63 Chang CF, Lin SZ, Chiang YH, Morales M, Chou J, Lein P, Chen HL, Hoffer BJ, Wang Y: Intravenous administration of bone morphogenetic protein-7 after ischemia improves motor function in stroke rats. Stroke 2003;34:558-564.

-64 Liu Y, Belayev L, Zhao W, Busto R, Saul I, Alonso O, Ginsberg MD: The effect of bone morphogenetic protein-7 (BMP-7) on functional recovery, local cerebral glucose utilization and blood flow after transient focal cerebral ischemia in rats. Brain Res 2001; 905:81-90.
65 Chou J, Harvey BK, Chang CF, Shen H, Morales $\mathrm{M}$, Wang Y: Neuroregenerative effects of BMP7 after stroke in rats. J Neurol Sci 2006;240:21-29.

66 Talbott JF, Cao Q, Enzmann GU, Benton RL, Achim V, Cheng XX, Mills MD, Rao MS, Whittemore SR: Schwann cell-like differentiation by adult oligodendrocyte precursor cells following engraftment into the demyelinated spinal cord is BMP-dependent. Glia 2006;54:147-159.

67 Zhang Z, Trautmann K, Artelt M, Burnet M, Schluesener HJ: Bone morphogenetic protein- 6 is expressed early by activated astrocytes in lesions of rat traumatic brain injury. Neuroscience 2006;138:47-53. 